\title{
Calibration Issues and Operating System Requirements for Electron-Probe Microanalysis
}

\author{
P. Carpenter* \\ *BAE SYSTEMS / NASA, Marshall Space Flight Center, AL 35812, USA
}

Instrument purchase requirements and dialogue with manufacturers have established hardware parameters for alignment, stability, and reproducibility, which have helped improve the precision and accuracy of electron microprobe analysis (EPMA). The development of correction algorithms and the accurate solution to quantitative analysis problems requires the minimization of systematic errors and relies on internally consistent data sets. Improved hardware and computer systems have resulted in better automation of vacuum systems, stage and wavelength-dispersive spectrometer (WDS) mechanisms, and x-ray detector systems which have improved instrument stability and precision. Improved software now allows extended automated runs involving diverse setups and better integrates digital imaging and quantitative analysis. However, instrumental performance is not regularly maintained, as WDS are aligned and calibrated during installation but few laboratories appear to check and maintain this calibration. In particular, detector deadtime (DT) data is typically assumed rather than measured, due primarily to the difficulty and inconvenience of the measurement process. This is a source of fundamental systematic error in many microprobe laboratories and is unknown to the analyst, as the magnitude of DT correction is not listed in output by microprobe operating systems.

Detector DT correction is made using the equation: $\mathrm{N}_{\mathrm{c}}=\mathrm{N}_{\mathrm{m}} /\left(1-\mathrm{N}_{\mathrm{m}} \tau\right)$, where $\mathrm{N}_{\mathrm{c}}$ is the correct x-ray intensity (in cps) without DT loss, $\mathrm{N}_{\mathrm{m}}$ is the measured intensity which has suffered DT loss, and $\tau$ is the DT constant [1]. This equation is rewritten: $\mathrm{N}_{\mathrm{m}} / \mathrm{i}=\mathrm{c}\left(1-\mathrm{N}_{\mathrm{m}} \tau\right)$, where $\mathrm{i}$ is the probe current, and $\mathrm{c}$ is a constant $\left(\mathrm{N}_{\mathrm{m}}=\mathrm{c} * \mathrm{i}\right)$, and is of the form $y=b+m x$, where $N_{m}$ is $x,\left(N_{m} / i\right)$ is $y$, and the y-intercept $b$ is the constant $c$. A plot of $N_{m}$ in cps vs. $\left(\mathrm{N}_{\mathrm{m}} / \mathrm{i}\right)$ in cps/nA is used (Fig. 1$)$, and $\tau$ is calculated from $\tau=\left[1-\left(\mathrm{N}_{\mathrm{m}} / \mathrm{i}\right) / \mathrm{c}\right] / \mathrm{N}_{\mathrm{m}}(\mathrm{i} . \mathrm{e} ., \tau=(1-\mathrm{y} / \mathrm{b}) / \mathrm{x})$. An Excel spreadsheet is used to calculate $\tau$ and to evaluate the integrity of the data set (Table 1). This spreadsheet has been interfaced to the Probe for Windows remote automation driver on the MSFC JEOL 8900 microprobe, and the DT measurement process is now fully automated. Each intensity measurement yields an experimental $\tau$ constant, and a least squares regression fit is made to two subsets of the intensity data. To identify nonlinear or paralyzable detector behavior, a large count rate range is used (i.e., $10-200 \mathrm{kcps}$ ). Charging of the target material may be a problem, and is monitored using the ratio of absorbed current to probe current. Both Si and Ti metal are used for DT measurements, as they provide high count rate intensities. Pulse measurements are performed in the integral mode due to significant pulse pileup at high count rates. Bias and pulse height analysis (PHA) scans are acquired over the range of count rates to study the pulse shift behavior of the counting system. Gain non-linearity and pulse shift at increased count rates result in erroneous measured $\tau$ values as pulse discrimination is lost. Strictly speaking, a DT measurement is only valid for the conditions at which the measurement was made, but extracted average values are used globally by the microanalysis community.

Older detector electronics exhibit $\tau$ from $\sim 2-5 \mu \mathrm{sec}$, and modern detector systems typically have $\tau \sim 1-1.5 \mu$ sec. An increase of $1 \mu \mathrm{sec}$ in $\tau$ represents a systematic error of $1 \%$ for each increase of $10 \mathrm{kcps}$, so a 3\% correction is implicit over the range $0-30 \mathrm{kcps}$ at $1 \mu \mathrm{sec}$, and $6 \%$ at $2 \mu \mathrm{sec}$. These errors exceed the quoted accuracy of microprobe analysis and underscore the need to minimize dependency on DT corrections and to match standard and sample count rates in addition to composition matching to minimize dependency on matrix correction. The low count rate range ( $<30 \mathrm{kcps}$ ) yields $\tau$ values significantly different compared to high count rate values and is thought to reflect dark current effects at low intensities (Fig. 2). The greatest apparent variability for $\tau$ is thus observed in the normal range of quantitative analysis. The dependence of experimental $\tau$ values on x-ray energy and count rate is not well known and is under continued study. The long term variation in $\tau$ is documented in Fig. 2, which illustrates the increase in $\tau$ with time due to progressive change in P-10 chemistry and/or detector aging. 
Instrument specification requirements of $1 \mu \mathrm{sec}$ deadtime on the Caltech JEOL 733 were met by installing new Noran PCS electronics which exhibited lower and more precise $\tau$ values.

The analyst must remain vigilant to deviations in instrumental alignment and calibration, and microprobe system software must conveniently verify the necessary parameters. Microanalysis of mission critical materials requires an ongoing demonstration of instrumental calibration. Possible approaches to improvements in instrument calibration, quality control, and accuracy will be discussed. Development of a set of core requirements based on discussions with users, researchers, and manufacturers can yield documents that improve and unify the methods by which instruments can be calibrated. These results can be used to continue improvements of EPMA.

\section{References}

[1] Heinrich, K.F.J. Electron Beam X-ray Microanalysis (1981)

\begin{tabular}{|c|c|c|c|c|c|c|c|c|c|}
\hline $\mathbf{n A}$ & \multicolumn{2}{|c|}{ Abs Cur Abs/Probe } & \multirow{2}{*}{$\begin{array}{c}\text { Time } \\
100\end{array}$} & \multirow{2}{*}{$\begin{array}{l}\text { Cps (x) } \\
4607.9\end{array}$} & \multirow{2}{*}{$\begin{array}{l}\text { Cps/nA (y } \\
230257\end{array}$} & \multirow{2}{*}{$\begin{array}{c}\text { Fit All } \\
2299.81\end{array}$} & \multirow[t]{2}{*}{ Fit Last } & \multicolumn{2}{|c|}{\begin{tabular}{|l|l|} 
DT us AII & DT Last \\
\end{tabular}} \\
\hline 2.00 & 1.63 & 0.82 & & & & & & 0.61 & \\
\hline 5.00 & 4.05 & 0.81 & 80 & 11436.9 & 2287.20 & 2286.17 & & 0.83 & \\
\hline 10.01 & 8.10 & 0.81 & 80 & 22665.3 & 2264.44 & 2263.73 & & 0.85 & \\
\hline 20.05 & 16.25 & 0.81 & 60 & 44469.0 & 2217.95 & 2220.18 & & 0.89 & \\
\hline 25.00 & 20.29 & 0.81 & 60 & 54977.1 & 2199.08 & 2199.18 & 2197.08 & 0.87 & 0.83 \\
\hline 29.98 & 24.34 & 0.81 & 30 & 65298.1 & 2177.91 & 2178.56 & 2177.00 & 0.87 & 0.84 \\
\hline 35.02 & 28.54 & 0.81 & 30 & 75474.9 & 2155.07 & 2158.23 & 2157.20 & 0.88 & 0.86 \\
\hline 40.05 & 32.53 & 0.81 & 30 & 85510.0 & 2134.97 & 2138.18 & 2137.67 & 0.88 & 0.86 \\
\hline 49.99 & 40.74 & 0.81 & 30 & 105026.6 & 2101.04 & 2099.19 & 2099.70 & 0.86 & 0.84 \\
\hline 69.98 & 57.25 & 0.82 & 30 & 141942.7 & 2028.45 & 2025.44 & 2027.88 & 0.86 & 0.84 \\
\hline \multicolumn{2}{|c|}{ Regression Output: } & & & & & \multicolumn{2}{|c|}{ Mean deadtime } & 0.86 & 0.84 \\
\hline All & \multicolumn{2}{|l|}{$Y$ intercept } & 2309.01 & Slope & -0.0020 & Sigma & & 0.02 & 0.01 \\
\hline High CR & \multicolumn{2}{|l|}{ Y intercept } & 2304.04 & Slope & -0.0019 & \multicolumn{2}{|c|}{ Regression DT } & 0.87 & 0.84 \\
\hline
\end{tabular}

Table 1. Excel spreadsheet used for deadtime calculation and remote automation with Probe for Windows operating system. X-ray intensity data are collected with probe and absorbed current. Least squares fit to Cps vs. $\mathrm{Cps} / \mathrm{nA}$ data is used to calculate deadtime constant $\tau$. Each discrete $\tau$ value can be compared to regression values.
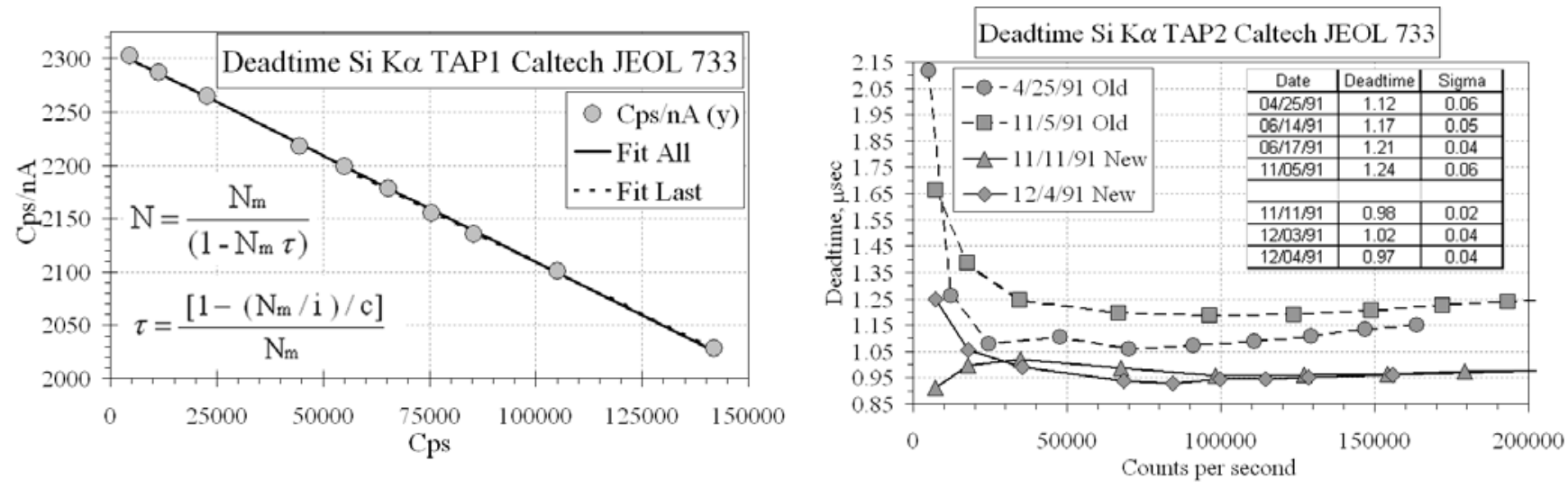

Fig. 1. (Left) Deadtime data for Si K $\alpha$ measurement exhibits excellent linearity and $\tau$ of $0.86 \pm 0.02 \mu$ sec using Noran PCS electronics that were developed to meet instrument specification for $\tau$ of $\leq 1 \mu \mathrm{sec}$.

Fig. 2. (Right) Variation of deadtime constant $\tau$ with count rate. DT values at low count rates are highly variable and not representative of high count rate regime. Note systematic increase in $\tau$ with time for older electronics, from 1.12 to $1.24 \mu \mathrm{sec}$, due to changing P-10 gas chemistry and/or detector aging. New electronics had $\tau$ of $\sim 1$ $\mu$ sec and $\sim 2 \mathrm{x}$ better precision. 